\title{
Verarbeitung von deutschen W-Fragen mit starken und schwachen Maskulina durch ein- und mehrsprachige Kinder und Erwachsene - Evidenz für morphosyntaktische Schemata?
}

\begin{abstract}
An der Schnittstelle von theoretischer Linguistik und Psycholinguistik erörtert dieser Beitrag zur Verarbeitung semantischer Rollen, ob und wie die mentale Repräsentation der sprachlichen Kodierung der Agens- und PatiensRollen im Rahmen gebrauchsbasierter Schema-Ansätze (Bybee 1985, 1988, 2010; Köpcke 1993) modelliert werden kann. Diese Fragestellung wird auf einer empirischen Datengrundlage diskutiert, die durch ein Eye Tracking Experiment (Visual World Paradigm) zur Verarbeitung von SVO- und OVS-Fragen mit starken und schwachen Maskulina durch kindliche L1- und L2-Lernende sowie erwachsene L1-Sprecherinnen und -Sprecher des Deutschen gewonnen wurde. Zentral ist die Frage, ob sich Argumente oder sogar Evidenzen dafür finden, dass Sprecherinnen und Sprecher in Abhängigkeit vom Grad der Spracherfahrung mit dem Deutschen in ihrer mentalen Grammatik morphosyntaktische Paar-Schemata (nach Wecker 2016) ausbilden, die sich gerade bei der Verarbeitung von starken und schwachen Maskulina, die ein unterschiedliches Deklinationsverhalten aufweisen, exemplarisch belegen lassen müssten.
\end{abstract}

\section{Einleitung}

In diesem Beitrag stellen wir die Ergebnisse eines Eye Tracking Experiments vor, mit dem wir an jüngere Erkenntnisse der psycholinguistischen Satzverarbeitungsforschung zur Interpretation von OVS-Strukturen im Deutschen anknüpfen.

Wertvolle Hinweise zu diesem Beitrag verdanken wir Verena Wecker, Jana Gamper sowie zwei anonymen Gutachten.

Anja Binanzer, Deutsches Seminar, Leibniz Universität Hannover

Valentina Cristante, Institut für Psycholinguistik und Didaktik der deutschen Sprache, Goethe-Universität Frankfurt am Main

Andreas Bittner, Deutsches Seminar, Leibniz Universität Hannover

Ә Open Access. (c) 2021 Anja Binanzer, et al., publiziert von De Gruyter. (cc) BY Dieses Werk ist lizensiert unter einer Creative Commons Namensnennung 4.0 International Lizenz. https://doi.org/10.1515/9783110710595-012 
Ausgangspunkt ist der Befund, dass bei solchen für das Deutsche atypischen Strukturen die Dekodierung des Patiens in satzinitialer Position bis $\mathrm{zu}$ einem Alter von ca. sieben Jahren noch nicht erfolgreich gelingt, da entsprechende morphologische Hinweise bei der Satzverarbeitung noch nicht hinlänglich berücksichtigt werden. Mit unserem Experiment wollen wir überprüfen, ob kindliche und auch erwachsene Sprecherinnen und Sprecher des Deutschen OVS-Strukturen dann erfolgreicher interpretieren, wenn die Patiens-Rolle morphologisch nicht nur am Artikelwort, sondern zusätzlich am Nomen gekennzeichnet wird. Dazu setzen wir in unserem Experiment in den zu verarbeitenden objekt-initialen W-Fragesätzen alternierend starke und schwache Maskulina ein, in denen das Patiens morphologisch entweder einfach (Welchen Mann küsst die Frau?) oder doppelt (Welchen Prinzen küsst die Frau?) gekennzeichnet ist.

Dieses experimentelle Setting erlaubt es darüber hinaus, der aus morphologietheoretischer Perspektive interessanten Frage nachzugehen, ob Sprecherinnen und Sprecher des Deutschen in ihrer mentalen Grammatik zur Repräsentation semantischer Rollen morphosyntaktische Schemata ausbilden. Am Beispiel der Verarbeitung von SVO- und OVS-Fragen mit starken und schwachen Maskulina kann insbesondere erörtert werden, ob sich diese Schemata mit zunehmender Spracherfahrung ausdifferenzieren und sie miteinander $\mathrm{zu}$ morphosyntaktischen PaarSchemata (Wecker 2016) verknüpft werden.

Der Beitrag ist wie folgt gegliedert: Nach einer knappen Darstellung, wie semantische Rollen im Deutschen grammatisch kodiert und in OVS-Strukturen verarbeitet werden (Abschnitte 2 und 3), stellen wir die gebrauchsbasierten SchemaModelle nach Bybee (1985, 1988, 2010), Köpcke (1993) und Wecker (2016) vor, auf deren Basis wir unseren Modellierungsvorschlag zur mentalen Repräsentation der Agens- und Patiens-Rollen als morphosyntaktische Schemata entwickeln (Abschnitt 4). Die daraus resultierenden Hypothesen (Abschnitt 5) werden schließlich auf ihre Plausibilität hin empirisch anhand der durch das Eye Tracking Experiment gewonnenen Daten überprüft (Abschnitte 6 und 7).

\section{Semantische Rollen im Deutschen: Grammatische Kodierung und Verarbeitung}

Mit semantischen Rollen werden in der Tradition der funktionalen Kasusgrammatik nach Fillmore (1968) die semantischen Relationen beschrieben, die zwischen einem Verb und den nominalen Elementen eines Satzes bestehen, wobei 
das Verb den nominalen Elementen seine semantischen Rollen zuweist. ${ }^{1}$ In transitiven Konstruktionen, die in diesem Beitrag behandelt werden, wie z. B. Die Oma küsst den Opa, fordert die Verbhandlung küssen ein diese Verbhandlung ausführendes Argument, das Agens (die Oma), und ein diese Verbhandlung erfahrendes Argument, das Patiens (den Opa).

Zur grammatischen Kodierung der Agens- und Patiens-Rollen stehen im Deutschen im Wesentlichen zwei Ebenen zur Verfügung: Syntax und Morphologie. ${ }^{2}$ Auf der syntaktischen Ebene kann die Wortstellung darüber Aufschluss geben, welches Argument eines transitiven Verbs die Agens- bzw. Patiens-Rolle einnimmt. So werden bei der sprachlichen Realisierung einer transitiven Verbhandlung Agens und Patiens in ein syntaktisches Muster überführt, in dem im Deutschen das Agens prototypischerweise die satzinitiale, präverbale, das Patiens die postverbale Position einnimmt (vgl. z. B. Primus 1999 oder Eisenberg 2013). Die Reihenfolge SVO (wie in dem oben angeführten Beispielsatz) wird deshalb auch als Basiswortstellung des Deutschen bezeichnet (vgl. Dryer 2013).

Semantische Rollen werden auf grammatischer Ebene nicht nur syntaktisch, sondern auch morphologisch kodiert. In unserem Beispielsatz zeigt neben der postverbalen Positionierung auch das dem maskulinen Substantiv Opa vorangehende Artikelwort den an, dass es sich bei der postverbalen NP um das Patiens handelt. Durch die Alternation der Artikelformen der vs. den im Singularparadigma der Maskulina ist die Kodierung der semantischen Rollen Agens oder Patiens also auch durch morphologische Kennzeichnung möglich.

Sowohl die syntaktische Positionierung als auch die morphologische Kennzeichnung geben im Deutschen aber nur mehr oder weniger bzw. nicht in jedem Fall zuverlässig Auskunft über die semantische Rolle eines Arguments. So kann das Patiens aufgrund der durch mehrere Faktoren ermöglichten relativ flexiblen Wortstellung des Deutschen ${ }^{3}$ auch satzinitial positioniert werden, z. B. Den Opa küsst die Oma (OVS). Die morphologische Eindeutigkeit der Rollen-Kodierung ist im Deutschen zudem genus- wie numerusabhängig. ${ }^{4}$ Nur das maskuline Paradigma im Singular zeigt die Agens- und Patiens-Rolle ein-

1 Vgl. dazu die kritische Diskussion in Ágel \& Höllein (i. d. B.).

2 Neben grammatischen Informationen werden auch kontextuelle, semantische und prosodische Mittel zur Kodierung von semantischen Rollen genutzt (vgl. ausführlich z. B. Gamper 2016). Wir gehen im Folgenden nicht weiter auf diese Aspekte ein und konzentrieren uns stattdessen auf die syntaktischen und morphologischen Mittel.

3 Vgl. die Charakterisierung der deutschen Wortstellung als „multifaktoriell“ z. B. Draye (2002).

4 Vgl. zum Zusammenhang der nominalen Kategorien aus systemlinguistischer und Erwerbsperspektive auch Bittner (i.d.B.) sowie Gamper, Wecker \& Szardenings (i.d.B.). 
deutig an (NOM: der vs. AKK: den), während das feminine und neutrale Paradigma an dieser Stelle synkretische Formen aufweisen (NOM: die, das vs. AKK: die, das).

Für die Verarbeitung von den für das Deutsche atypischen OVS-Strukturen mit einem morphologisch eindeutig ausgewiesenen Patiens in satzinitialer Position wurde in verschiedenen Studien mit monolingual deutschsprachig aufgewachsenen Kindern (L1) und Kindern, die Deutsch sukzessiv als frühe Zweitsprache (L2) erwerben, gezeigt, dass sie sich bis zu einem Alter von ca. sieben Jahren stärker an der syntaktischen als an der morphologischen Information orientieren (vgl. zuletzt z. B. die Bildauswahlstudien von Roesch \& Chondrogianni 2014, 2015, 2016; die Eye Tracking-Studien von Cristante 2016; Cristante \& Schimke 2018 oder Schouwenaars, Hendriks \& Ruigendijk 2018, die EEG-Studie von Schipke et al. 2012; die MRI-Studie von Fengler, Meyer \& Friederici 2015 oder die Self Paced Reading Studie von Gamper 2016). Demnach wird im Vor- und Grundschulalter der satzinitialen NP präferiert die Agens-Rolle zugewiesen, auch wenn die NP am Artikelwort morphologisch eindeutig als Patiens kodiert ist. Diese Satzinterpretationsstrategie wird entsprechend als ,agent first-Strategie‘ bezeichnet (vgl. Bever 1970). Für kompetente erwachsene Sprecherinnen und Sprecher des Deutschen, die in manchen der Studien als Kontrollgruppen hinzugezogen wurden, zeigte sich hingegen, dass sie OVS-Strukturen richtig interpretieren können, wenn sie morphologische Hinweise zur Verteilung der semantischen Rollen enthalten. Für die ontogenetische Entwicklung von Dekodierungsfähigkeit syntaktischer und morphologischer Informationen lässt sich den Ergebnissen oben genannter Studien nach zusammenfassen, dass zunächst eine syntaktisch gesteuerte Interpretationsstrategie angewendet wird (agent first-Strategie) und morphologisch basierte Interpretationsfähigkeiten erst darauffolgend entwickelt werden. ${ }^{5}$

5 Im Rahmen des gebrauchsbasierten Competition Models (MacWhinney \& Bates 1989; MacWhinney 2001, 2017) kann dieser Befund dadurch erklärt werden, dass sich die Gewichtung der syntaktischen und morphologischen Informationen bei der Satzinterpretation mit zunehmender Spracherfahrung verändert und entsprechend unterschiedliche Strategien der Satzinterpretation entwickelt und angewendet werden. Dabei beeinflussen auch die Frequenz und die Reliabilität der sprachlichen Hinweisreize die Entwicklung dieser Strategien. Der (L1- oder L2-)Spracherwerb wird in diesem Modell als ein Prozess konzeptualisiert, in dem Sprecherinnen und Sprecher Formen und Strukturen einer zu erwerbenden Sprache aus dem Input abstrahieren und ihnen dabei semantische und grammatische Funktionen zuweisen (,form function mapping). Um Form-Funktions-Relationen herstellen zu können, müssen sog. ,cues‘ der zu erwerbenden Sprache erkannt werden, worunter funktionsindizierende Hinweise einer Sprache zu verstehen sind. Bezogen auf die rezeptive Dekodierung bzw. produktive Markierung semantischer Rollen im Deutschen zählen 


\section{Morphologische Doppelmarkierung}

Eine $u$.W. empirisch noch nicht überprüfte Frage ist, ob die Verarbeitung von OVS-Strukturen positiv beeinflusst wird, wenn die morphologische Markierung verdoppelt wird. ${ }^{6}$ M.a.W. fragen wir danach, ob objekt-initiale Strukturen mit einer morphologisch mehrfach als Patiens ausgewiesenen NP besser als nichtagentivisch dekodiert werden können als einfache, d. h. nur durch das Artikelwort markierte objekt-initiale Strukturen. Diese Konstellation ist im Deutschen im Kontext von schwachen Maskulina gegeben. So besteht für die Deklinationsklasse der schwachen Maskulina die Notwendigkeit, die Patiens-Rolle in einfachen NP, bestehend aus Artikelwort und Substantiv, doppelt zu kennzeichnen, nämlich am Artikelwort sowie durch die obligatorische Suffigierung eines Kasusflexivs am Substantiv selbst. Dieses morphologische Charakteristikum unterscheidet die schwachen von den starken Maskulina, vgl. ihr unterschiedliches Flexionsverhalten im NOM und AKK Singular in Tabelle 1. Mit dem Blick auf das Primat der gesprochenen Sprache im Spracherwerb sowie auf den in unserem Experiment ausschließlich auditiv zu verarbeitenden Input enthält die Tabelle neben der traditionell graphematischen Darstellung der Beispiele auch phonologische Transkriptionen.

dazu u. a. die beschriebenen syntaktischen und morphologischen Mittel. Ihr (produktiver wie rezeptiver) Erwerb ist wiederum von der Validität der cues abhängig. Die cue-Validität setzt sich aus der ,cue availability، (seiner Frequenz) im sprachlichen Input und seiner ,reliability، (Vorkommen des cues, in dem er tatsächlich die entsprechende Funktion erfüllt) zusammen. Die Validität eines cues, die sich objektiv durch eine Systemanalyse ermitteln lässt, wie sie beispielsweise Kempe \& MacWhinney (1998) durch eine Korpusanalyse von Lehrwerken für erwachsene Lernerinnen und Lerner des Deutschen als Fremdsprache oder Dittmar, Abbot-Smith, Lieven \& Tomasello (2008) durch die Analyse kindgerichteter Sprache für die Validität morphologischer und syntaktischer cues durchführten, muss aber nicht mit der Validität eine cues in der (ontogenetischen) Sprachentwicklung übereinstimmen. Entsprechend wird im Competition Model auch zwischen der objektiven und subjektiven cue-Validität (,overall cue validity' und ,cue strength') unterschieden. So fasst etwa Gamper (2016: 95-102) für einen Vergleich verschiedener Satzverarbeitungsstudien mit Kindern zusammen, dass diese im Verlauf des Spracherwerbs „[. . .] sehr systematisch einen Justierungsprozess [durchlaufen], in dem die ursprünglich hohe Validität eines cues zugunsten eines anderen aufgegeben wird“.

6 Zwar setzten Roesch \& Chondrogianni (2014) in einer Studie zur Verarbeitung von deutschen SVO- und OVS-Fragesätzen durch kindliche L1-, simultan zweisprachig aufgewachsene (2L1) und frühe sukzessive L2-Lernende im Alter zwischen 5,0 und 5,11 Jahren sowie durch erwachsene L1-Sprecherinnen und -Sprecher schwache Maskulina in ihrem Testset ein; allerdings überprüften sie diese Struktur nur durch SVO-Fragen mit doppelt markierten maskulinen NPs in postverbaler Position (Welcher Elefant malt den Bären an?). Ob doppelt markierte schwache Maskulina auch in OVS-Fragesätzen zu einer erfolgreicheren Verarbeitung führen, wurde in dieser Studie aber nicht untersucht. 
Tab. 1: Deklinationsparadigmen starker und schwacher Maskulina im Singular.

\begin{tabular}{lll}
\hline $\begin{array}{l}\text { Flexion } \\
\text { Singular }\end{array}$ & $\begin{array}{l}\text { starke Maskulina } \\
\text { Hund, Tiger, Arzt, Bäcker . . . }\end{array}$ & $\begin{array}{l}\text { schwache Maskulina } \\
\text { Prinz, Bär, Löwe, Junge . . . }\end{array}$ \\
\hline Nom & $\langle$ der Hund $>$ [dee hont] & $<$ der Prinz> [dee prınts] \\
Akk & $<$ den Hund [den hont] & $<$ den Prinzen> [den prıntsñ] \\
\hline
\end{tabular}

Der Akkusativ wird bei schwachen Maskulina im Singular mittels eines Suffixes kodiert, bei auf Schwa auslautenden schwachen Maskulina (des Typs Löwe, Junge) wird die Schwasilbe mittels <- $n>$ geschlossen, andernfalls erfolgt die Suffigierung von<-en> (bei schwachen Maskulina des Typs Bär, Prinz). Der oblique Kasus wird also entweder additiv silbisch (-en) oder (bei auf Schwa auslautender zwei- oder mehrsilbiger Struktur) silbenschließend $(-n)$ gekennzeichnet. $^{7}$

Da wir vor dem Hintergrund der in Abschnitt 3 detaillierter vorzustellenden Schema-Modelle (Bybee 1985, 1988, 2010; Köpcke 1993; Wecker 2016) davon ausgehen, dass die Frequenz sprachlicher Strukturen im sprachlichen Input den Spracherwerb und die Sprachverarbeitung beeinflusst, muss auch danach gefragt werden, in welcher Häufigkeit schwache Maskulina auftreten. Laut der Berechnung von Köpcke (1995: 160) stellen die schwachen Maskulina bezogen auf den gesamten nominalen Wortschatz (Maskulina, Feminina, Neutra) mit einer Typefrequenz von 2,5\% eine „relativ kleine“ Deklinationsklasse dar. Bezogen auf ihre relative Frequenz im Vergleich zu stark und gemischt deklinierenden Maskulina führt Ronneberger-Sibold (i.d.B.) auf der Basis des Dudens (2016: 219-220) die in Tabelle 2 dargestellten Werte an (vgl. ebenda auch die Erläuterung zur Zusammensetzung der Werte).

Tab. 2: Relative Frequenz stark, schwach und gemischt deklinierter Maskulina nach Ronneberger-Sibold (i. d. B.) / Duden (2016).

\begin{tabular}{lrrr}
\hline & \multicolumn{1}{l}{ starke Maskulina } & \multicolumn{1}{c}{ schwache Maskulina } \\
& Hund, Tiger, Arzt, Bäcker & Bär, Prinz, Löwe, Junge & $\begin{array}{l}\text { gemischte Maskulina } \\
\text { Staat, Vetter, Ende, Fleck }\end{array}$ \\
\hline Types & $66,4 \%$ & $17,4 \%$ & $3 \%$ \\
Tokens & $76,6 \%$ & $12,5 \%$ & $2,5 \%$ \\
\hline
\end{tabular}

$7 \mathrm{Zu}$ den Bedingungen und zur Funktionalität der spezifischen Entwicklung der Flexionsklasse der schwachen Maskulina im Deutschen vgl. auch Ronneberger-Sibold (i.d.B.); zur davon abweichenden Entwicklung im Schwedischen und Dänischen vgl. außerdem Kürschner (i.d.B.). 
Es wird also deutlich, dass die schwachen Maskulina verglichen mit den starken und gemischten Maskulina eine im Input nicht besonders frequent vertretene Klasse sind.

Hinzu kommt, dass für das Deklinationsverhalten einiger schwacher Maskulina gegenwärtig Sprachwandelprozesse zu beobachten sind. Einer dieser Prozesse betrifft den Abbau der Markierung der obliquen Kasus am Substantiv selbst (dem/den *Bär, Prinz, Mensch), d. h. dass schwache Maskulina gänzlich oder teilweise wie starke (oder gemischte, vgl. der Glaube/Friede > der Glauben/ Frieden) flektiert werden (vgl. etwa Thieroff 2003; Köpcke 2005; Paulfranz 2013) und die Patiens-Rolle somit nicht (mehr durchgängig) doppelt markiert wird.

Betrachten wir aber die in unserem Experiment eingesetzten starken und schwachen Maskulina hinsichtlich ihrer Frequenz im kindlichen rezeptiven wie produktiven Wortschatz, ergibt sich ein anderes Bild. In Tabelle 3, in der die Testitems mit ihren Tokenfrequenzwerten nach childLex (Schroeder et al. 2015) sowie Pregel \& Rickheit (1987) angegeben sind, stellen wir die Frequenzwerte des anzunehmenden rezeptiven und produktiven nominalen Lexikons von deutschsprachigen Kindern im Grundschulalter einander gegenüber. childLex ist ein Korpus, das auf einer repräsentativen und aktuellen Auswahl von 500 Büchern für Kinder und Jugendliche im Alter von sechs bis zwölf Jahren mit insgesamt ca. zehn Millionen Wörtern basiert. Damit ist es repräsentativ für den anzunehmenden sprachlichen Input (literaler, geschriebener Sprache), auf dessen Grundlage sprachliche Muster abstrahiert werden können. Das Frequenzwörterbuch von Pregel \& Rickheit (1987) umfasst demgegenüber lediglich 260.000 Wörter, ist aber u. W. noch immer das aktuellste Korpus zum produktiven Wortschatz im Grundschulalter.

Während wir für das Auftreten der Testitems in den unterschiedlichen Kasus (im SG) für childLex anhand von Kontexttrefferlisten selbst Frequenzanalysen vornehmen konnten, ${ }^{8}$ sind dem Korpus von Pregel \& Rickheit (1987) diesbezügliche Differenzierungen nicht zu entnehmen. Im Gegensatz zu den nach Ronneberger-Sibold (i.d. B.) bzw. Duden (2016) ermittelten Frequenzwerten zei-

8 Wir danken Sascha Schroeder für das Erstellen der Kontexttrefferlisten und seine Bereitschaft, die childLex-Daten auf diese Weise für unsere Untersuchung zugänglich zu machen. Analysiert wurden alle Kontexttreffer hinsichtlich ihres Vorkommens in den unterschiedlichen Kasus und Numeri, wobei die Analyse nicht auf ausschließlich transitive Konstruktionen eingegrenzt wurde. Komposita, in denen die Substantive als Letztglied fungierten, wurden berücksichtigt. Von der Analyse wurden solche Belege ausgeschlossen, in denen die Substantive als Eigennamen (z. B. Graf Runkelstein), zur Anrede (Junge, sei vernünftig!) oder als Ortsangabe (Siehst du den Bäcker?) verwendet wurden. Ambig interpretierbare Kontexttreffer wurden ebenfalls ausgeschlossen. 
Tab. 3: Im Experiment verwendete starke und schwache Maskulina unter Angabe ihrer Tokenfrequenz im rezeptiven (childLex, vgl. Schroeder et al. 2015) und produktiven kindlichen Wortschatz (Pregel \& Rickheit 1987).

\begin{tabular}{|c|c|c|c|c|c|}
\hline \multirow[b]{2}{*}{$\begin{array}{l}\text { starke Maskulina } \\
\text { (SG) }\end{array}$} & \multicolumn{4}{|c|}{$\begin{array}{l}\text { rezeptiver Wortschatz } \\
\text { childLex (2015) }\end{array}$} & \multirow[t]{2}{*}{$\begin{array}{l}\text { produktiver Wortschatz } \\
\text { Pregel \& Rickheit (1987) }\end{array}$} \\
\hline & $\begin{array}{r}\text { NOM } \\
\varnothing\end{array}$ & $\begin{array}{r}\text { AKK } \\
\varnothing\end{array}$ & $\begin{array}{r}\text { DAT } \\
\varnothing\end{array}$ & $\begin{array}{l}\text { GEN } \\
-(e) s\end{array}$ & \\
\hline Dieb & 191 & 70 & 20 & 6 & 0 \\
\hline Wolf & 188 & 44 & 41 & 20 & 20 \\
\hline Fuchs & 162 & 30 & 32 & 4 & 54 \\
\hline Arzt & 148 & 31 & 74 & 6 & 76 \\
\hline Tiger & 108 & 9 & 12 & 4 & 30 \\
\hline Maler & 28 & 2 & 2 & 2 & 0 \\
\hline Bäcker & 26 & 5 & 39 & 1 & 6 \\
\hline Panter & 2 & 4 & 0 & 0 & 0 \\
\hline$\Sigma \mathrm{N} 1.311$ & 853 & 195 & 220 & 43 & 186 \\
\hline in $\%$ & 65,1 & 14,9 & 16,8 & 3,3 & \\
\hline $\begin{array}{l}\text { schwache Maskulina } \\
\text { (SG) }\end{array}$ & $\begin{array}{r}\text { NOM } \\
\varnothing\end{array}$ & $\begin{array}{l}\text { AKK } \\
-(e) n\end{array}$ & $\begin{array}{l}\text { DAT } \\
-(e) n\end{array}$ & $\begin{array}{l}\text { GEN } \\
-(e) n\end{array}$ & \\
\hline Junge & 1.839 & 485 & 328 & 93 & 225 \\
\hline Bär & 369 & 81 & 63 & 20 & 0 \\
\hline Prinz & 289 & 65 & 51 & 27 & 16 \\
\hline Riese & 158 & 41 & 40 & 34 & 0 \\
\hline Hase & 139 & 47 & 28 & 3 & 79 \\
\hline Affe & 110 & 23 & 31 & 4 & 135 \\
\hline Graf & 60 & 11 & 11 & 26 & 0 \\
\hline Spatz & 29 & 2 & 2 & 1 & 8 \\
\hline$\Sigma N 4.510$ & 2.993 & 755 & 554 & 208 & 463 \\
\hline in $\%$ & 66,4 & 16,7 & 12,3 & 4,6 & \\
\hline
\end{tabular}

gen die Daten aus childLex ein anders gelagertes Frequenzverhältnis der starken und schwachen Maskulina für den anzunehmenden rezeptiven und produktiven kindlichen Wortschatz, zumindest unsere Testitems betreffend. Die von uns für das Experiment nach den Kriterien Mensch/Tier und ein-/zweisilbig ausgewählten schwachen Maskulina treten demnach sowohl im rezeptiven als auch im produktiven nominalen Lexikon von Grundschulkindern wesentlich häufiger als die von uns verwendeten starken Maskulina auf.

Um die tatsächliche relative Frequenz der beiden Deklinationsklassen feststellen zu können, wäre eine Frequenzberechnung aller in beiden Korpora verzeichneten starken und schwachen Maskulina notwendig (und nicht nur jene 
unserer Testitems). Die für unsere Testitems angeführten Frequenzwerte erachten wir aber auch unabhängig von einer solchen Berechnung als Beleg dafür, dass zumindest bestimmte Vertreter der jeweiligen Deklinationsklasse im potentiellen kindlichen Input/Output eine hohe Tokenfrequenz aufweisen. Folglich gehen wir auch davon aus, dass das Flexionsmuster der schwachen Maskulina trotz der Tatsache, dass es sich bei dieser Deklinationsklasse im allgemeinen Wortschatz um eine kleine Typeklasse mit insgesamt niedriger Tokenfrequenz handelt, als im kindlichen Input verfügbar vorausgesetzt werden kann. Eine experimentelle Untersuchung von Indefrey (2002: 67) zeigt zudem, dass einsprachig aufgewachsene Kinder im Alter von fünf Jahren ihnen bekannte schwache Maskulina im AKK und DAT zielsprachlich flektieren und im Alter von acht Jahren die schwache Deklination auch produktiv auf Kunstwörter anwenden können. Mit dem Blick auf ein Sprachverarbeitungsexperiment von Casenhiser und Goldberg (2005) mit fünf- bis siebenjährigen Kindern nehmen wir zudem an, dass der Aufbau von sprachlichen Form-Funktions-Relationen insbesondere dann positiv unterstützt wird, wenn ein Type einer bestimmten Klasse eine hohe Tokenfrequenz aufweist, wie es z. B. auf unser Testitem Junge zutrifft, d. h. Musterbildung bereits auf der Basis einzelner prototypischer Vertreter möglich ist. Im von Grießhaber (i.d.B.) analysierten Korpus schriftsprachlicher Texte von ein- und mehrsprachigen Grundschulkindern des 2. und 4. Jahrgangs zeigt sich in der Detailanalyse zur Verwendung des schwachen Maskulinums Junge auch, dass es die einsprachigen Kinder bereits „fehlerfrei“ verwenden. Nur bei den mehrsprachigen Grundschulkindern treten Abweichungen auf, worunter die Verwendung des nicht flektierten Substantivs in Akkusativ- und Dativphrasen fällt.

Addiert man die Werte der unterschiedlichen im Singularparadigma vorhandenen Wortformen, die Rückschlüsse auf ihre Funktionen zulassen, zeigt sich für die starken Maskulina, dass zu 96,8\% die gleiche Wortform auftritt (in den drei Kasus NOM, AKK und DAT). Da die Wortform für die Agens- und die Patiens-Rolle die gleiche morphologische Gestalt aufweist, kann von ihr selbst keine Funktion abgeleitet werden; stattdessen kodiert nur das Artikelwort die semantische Rolle. Demgegenüber steht bei den schwachen Maskulina ein Verhältnis von 66,4\% für den NOM zu 33,6\% für die obliquen Kasus. Die AgensRolle wird hier durch den (endungslosen) NOM also eindeutig kodiert. Zwar lässt die synkretische Wortgestalt [Xen] in den obliquen Kasus der schwachen Maskulina auch nicht eindeutig auf die Patiens-Rolle schließen, eine [Xen]-Gestalt in einer transitiven Konstruktion im Singular kann aber nur als Patiens interpretiert werden. Außerdem stellt der AKK gegenüber dem DAT und dem GEN den Kasus mit der höchsten Frequenz dar.

Bezogen auf unsere Fragestellung kann somit angenommen werden, dass die morphologisch deutlichere Patiens-Markierung der schwachen Maskulina 
bei der Satzverarbeitung zu einer erfolgreicheren Interpretation führt; die niedrigen Frequenzwerte der schwachen Maskulina im allgemeinen Wortschatz und die Sprachwandelprozesse, von denen sie betroffen sind, könnten allerdings vermuten lassen, dass es sich bei der Doppelmarkierung von schwachen Maskulina um eine insgesamt so selten auftretende Struktur handelt, dass sich keine begünstigenden Einflüsse auf die Satzverarbeitung ergeben. Da aber für den kindlichen Wortschatz (exemplarisch an unseren Testitems) gezeigt werden kann, dass schwache Maskulina darin eine hohe Tokenfrequenz aufweisen, und einsprachig aufwachsende Kinder ab einem Alter von fünf Jahren laut der Studie von Indefrey (2002) auch damit beginnen, das schwache Deklinationsmuster produktiv anzuwenden, ist davon auszugehen, dass dieses sprachliche Muster bereits im Kindesalter aus dem Input abstrahiert wird und sich somit die Frequenz des Musters im kindlichen Wortschatz und die ,auffällige‘ Doppelmarkierung bei der Satzverarbeitung gegenseitig positiv unterstützen.

\section{Semantische Rollen und Schema-Ansätze}

Die aus morphologietheoretischer Sicht interessante Frage zur mentalen Repräsentation der sprachlichen Kodierung semantischer Rollen, der wir mit unserem Experiment v. a. nachgehen wollen, ist von Ergebnissen der Untersuchung von Wecker (2016) zum produktiven Erwerb der deutschen Pluralmorphologie durch kindliche L2-Lernende des Deutschen inspiriert. Vor dem Hintergrund gebrauchsbasierter Schema-Modelle von Bybee (1985, 1988, 2010) und Köpcke (1993) gelingt es Wecker zu zeigen, dass die von ihr untersuchten L2-Lernenden auf dem Weg zur zielsprachlichen Pluralbildung zunächst einfache Singularund Plural-Schemata ausbilden, die mit zunehmender Spracherfahrung auf der Basis ihrer paradigmatischen Beziehungen zu Paar-Schemata verknüpft werden. In unserem Beitrag soll diese Modellierung für die deutsche Kasusmorphologie, d.h. hier die morphologische Kodierung von semantischen Rollen in Mehrworteinheiten (NP bestehend aus Artikelwort und Substantiv), fruchtbar gemacht werden. Dazu skizzieren wir zunächst die Grundgedanken gebrauchsbasierter Schema-Modelle und die in diesem theoretischen Rahmen von Köpcke (1993) vorgenommene Modellierung der deutschen Pluralmorphologie, um daran anschließend etwas detaillierter Weckers (2016) Vorschlag zur Ausbildung von Paar-Schemata vorzustellen. Schließlich unterbreiten wir unsere Überlegungen zur mentalen Repräsentation der Agens- und Patiens-Rolle als morphosyntaktische Paar-Schemata. 


\subsection{Morphologische Schemata}

Morphologische Schemata werden in den gebrauchsbasierten Modellen von Bybee (1985, 1988, 2010), Köpcke (1993) oder Wecker (2016) als abstrakte, dynamische kognitive Einheiten definiert, die Sprecherinnen und Sprecher im Verlauf des Spracherwerbs bzw. mit zunehmender Spracherfahrung durch Vergleichs-, Kategorisierungs- und Abstraktionsprozesse in ihrer mentalen Grammatik ausbilden. Der Aufbau morphologischen Wissens vollzieht sich demnach als ein integratives Zusammenspiel von Lexikon und Grammatik, zumal davon ausgegangen wird, dass Sprecherinnen und Sprecher zwischen im mentalen Lexikon zunächst holistisch gespeicherten - einfachen wie komplexen - Wortformen Verknüpfungen herstellen, wenn diese semantische und phonologische Ähnlichkeiten aufweisen. Beim Vergleich dieser Einzelexemplare werden auf der Basis ihrer Gemeinsamkeiten in Form und Funktion auf einer abstrakteren Ebene Schemata für Form-Funktions-Relationen abgeleitet, die wiederum in einem assoziativen Netzwerk miteinander verbunden sind. Anders als in strikt formalistischen, regelbasierten Modellen vollzieht sich der Aufbau morphologischen Wissens in diesen Modellierungen also durch gebrauchsbasierte Generalisierungsprozesse auf der Basis des zur Verfügung stehenden sprachlichen Inputs als ein ,bottom up“Prozess, die Grammatik geht im Lexikon auf.

Köpcke (1993) entwickelt auf der Basis dieser Grundannahmen eine schemabasierte Modellierung ${ }^{9}$ der deutschen Pluralmorphologie. Als Singular-/Pluralschemata definiert Köpcke (1993: 10) ,abstrakte, vom Sprecher des Deutschen gespeicherte Gestalten [. . .], die mit den Funktionen Singular und Plural kompatibel sind“. Zentral ist hier, dass konkrete Singular- und Pluralwortformen im mentalen Lexikon zunächst als holistische ,Gestalten' gespeichert sind, dann auf der Grundlage von semantischer und/oder phonotaktischer Identität miteinander verknüpft und in einem nächsten Schritt auf abstrakter Ebene zu Schemata für die grammatischen Funktionen Singular bzw. Plural zusammengefasst und kognitiv repräsentiert werden. Auf diese abstrakten (von der konkreten Wortform losgelösten) Schemata wird dann bei der Sprachverarbeitung oder -produktion zugegriffen. Der wesentliche Unterschied dieser Modellierung zu regelbasierten Modellen besteht entsprechend darin, dass komplexe Wortformen wie Pluralformen nicht durch die Segmentierung bzw. Kombination von Wurzel- und grammatischen Morphemen dekodiert bzw. generiert werden müssen, da sie nicht von Singularformen abgeleitet, sondern in ihrer

9 Sein Ansatz versteht sich als Synthese von regelbasierten Ansätzen und dem diesen Modellen konträr gegenüberstehenden Entwurf von Bybee (1985), in dem grammatische Regelkomponenten vollständig im Lexikon aufgehen. Kritische Anmerkungen dazu finden sich in Wecker (2016: 44-46). 
Gestalt wie Singularformen selbstständig repräsentiert werden. Die Singularund Plural-Schemata sind auf der Grundlage ihrer semantischen und formalen Ähnlichkeiten überdies in einem assoziativen Netzwerk miteinander verbunden. Es bestehen also Verknüpfungen sowohl zwischen Singular- und Pluralschemata als auch zwischen unterschiedlichen Singular- bzw. Pluralschemata untereinander.

Ob Sprecherinnen und Sprecher Wortformen als Singular- oder Pluralformen klassifizieren, ist nach Köpcke (1993: 82-85) von der ,Signalstärke‘ (,cue strength' nach dem Competition Model von Bates \& MacWhinney 1989) einer Wortgestalt, eine der beiden grammatischen Funktionen $\mathrm{zu}$ indizieren, abhängig. Die Signalstärke einer Wortgestalt für eine Funktion setzt sich wiederum aus verschiedenen Komponenten zusammen, von denen insbesondere die Type- bzw. Tokenfrequenz sowie die Signalvalidität der Wortformen für eine bestimmte grammatische Funktion hervorzuheben sind. So beschreibt Köpcke (1993: 82-85) Wortformen des Typs [\#_en] (wie Frauen oder Wiesen) als besonders signalstarke Gestalten für die Funktion Plural, da die Wortgestalt [\#_en] in der Funktion Plural frequenter ist als andere Plural indizierende Wortgestalten (wie z. B. [\#_er] für Kinder oder [\#_e] für Tische) und aufgrund der nur wenigen Wortformen, bei denen die [\#_en]-Wortgestalt in der Funktion Singular auftritt (wie Garten oder Rasen), valider die Funktion Plural indiziert. Aus dem Vergleich der Signalstärke unterschiedlicher potentieller Pluralgestalten leitet Köpcke ein Kontinuum mit graduellen Abstufungen für prototypische Plural- bzw. prototypische Singulargestalten ab, die in der mentalen Grammatik wiederum als Singular- oder Pluralschemata repräsentiert anzunehmen sind.

\subsection{Paar-Schemata nach Wecker (2016)}

Das soeben knapp skizzierte Schema-Modell Köpckes zur deutschen Pluralmorphologie validiert Wecker (2016) empirisch durch eine Untersuchung mit kindlichen Lernenden des Deutschen als Zweitsprache. Wecker zeigt, dass die von ihr untersuchten Kinder zur Erschließung des deutschen Pluralsystems im Verlauf ihres Zweitspracherwerbs Singular- und Pluralschemata ausbilden. ${ }^{10}$ Für diesen sich in unterschiedlichen Phasen vollziehenden Erwerbsprozess beobachtet sie die Entwicklung und Anwendung unterschiedlicher Strategien, bei denen sie produkt- und source-orientierte Strategien unterscheidet und die be-

10 Vgl. auch Gamper, Wecker \& Szardenings (i.d. B.). 
zogen auf die Ausbildung von Singular- und Pluralschemata wie folgt definiert sind:

Eine Pluralbildung, die unabhängig von der zugehörigen Singularform im Abgleich mit dem prototypischen Pluralschema vorgenommen wird, ist produktorientiert. Wird jedoch ein Plural gebildet, der die Paarbeziehung zwischen Singular- und Pluralschemata (also Paar-Schemata) berücksichtigt, liegt eine source-orientierte Pluralbildung vor.

(Wecker 2016: 59)

Produktorientierte Pluralbildungen basieren demnach auf der Schemaabstraktion von Pluralgestalten, source-orientierte Pluralbildungen dagegen auf den Beziehungen, die zwischen Singular- und Pluralschemata bestehen, den sog. ,Paar-Schemata‘. Hierunter sind dezidiert nicht regelbasierte Ableitungen der Pluralformen von Singularformen (source) zu verstehen. Stattdessen wird diese Strategie in Anknüpfung an Nessets (2008) ,first-order schemas“ und ,second-order schemas` modelliert. Mit einem first-order schema wird eine „Generalisierung über Formen, die die gleiche Funktion bzw. Bedeutung tragen“ (Wecker 2016: 59), definiert. Ein solches einfaches, first-order Schema wie es z. B. das prototypische Pluralschema [\#_en] darstellt, wird demnach auf der Basis von ihm entsprechenden Wortformen abgeleitet, die die Funktion Plural transportieren (wie Frauen, Wiesen, Blumen). Second-order schemas werden dagegen bezogen auf die paradigmatischen Verbindungen zwischen solchen einfachen Schemata etabliert. Für das Pluralschema [\#_en] bedeutete dies, dass es demnach mit dem Singularschema [\#_e] verknüpft wird (vor dem Hintergrund, dass im Singular auf Schwa auslautende Substantive wie Wiese, Junge etc. im Plural der Wortgestalt [\#_en] entsprechen).

Mit dieser Modellierung wird der für Schema-Ansätze zentrale Gedanke zur Organisation des gesamten mentalen Lexikons einer Sprecherin / eines Sprechers in Form eines Netzwerks auf der Ebene der paradigmatischen Beziehungen noch weiter ausbuchstabiert. Entsprechend bestehen nicht nur zwischen einzelnen Wortgestalten und Funktionen Verknüpfungen, von denen einfache Schemata abgeleitet werden; vielmehr werden einfache Schemata mit zunehmender Spracherfahrung auch miteinander zu Paar-Schemata verknüpft. Die von Wecker detektierten Strategien bei der Pluralbildung können wiederum zunächst auf die Ausbildung einfacher Schemata, später auf das Vorhandensein von Paar-Schemata zurückgeführt werden. 


\subsection{Paar-Schemata zur Repräsentation der Agens- und Patiens-Rolle}

Adaptieren wir zunächst die Modellierung von Köpcke (1993) auf die mentale Repräsentation der Agens- und Patiens-Rolle, lässt sich ableiten, dass Sprecherinnen und Sprecher durch die (wiederholte) Verarbeitung transitiver Singular-Konstruktionen von den darin auftretenden (einfachen) NP, bestehend aus Artikelwort und einem maskulinen Substantiv, wie der Hund, der Tiger (starke Maskulina) bzw. der Prinz, der Löwe (schwache Maskulina) etc. ein morphosyntaktisches Agens-Schema des Typs [der X] abstrahieren. Dieses ist für starke und schwache Maskulina identisch. Für die Patiens-Rolle muss aufgrund des unterschiedlichen Deklinationsverhaltens starker und schwacher Maskulina angenommen werden, dass das Patiens-Schema binär ausdifferenziert wird, da als Wortgestalten zwei Typen zur Verfügung stehen: [den X] für Akkusativ-Phrasen mit starken Maskulina wie den Hund, den Tiger und [den Xen] für Akkusativ-Phrasen mit schwachen Maskulina wie den Prinzen, den Löwen. ${ }^{11}$

Betrachtet man nur die Wortgestalt der starken und schwachen Maskulina unabhängig vom Artikelwort, kann hinsichtlich ihrer Signalvalidität eindeutig bestimmt werden, dass das von schwachen Maskulina abstrahierte Schema [den Xen] signalstärker als das von starken Maskulina abstrahierte Schema [den X] ist. Die Wortgestalt [Xen] kann in transitiven Singular-Konstruktionen nur im Fall von auf -en auslautenden Simplizia wie Garten oder Rasen in der Agens-Rolle auftreten und stellt ansonsten ein exklusives Schema für die Patiens-Rolle dar. Bei starken Maskulina unterscheidet sich die nominale Wortgestalt [X] in ihrem Auftreten in der Funktion Agens oder Patiens nicht, sodass deshalb angenommen werden kann, dass sie mit beiden Funktionen gleichermaßen stark assoziiert ist. Sie weist entsprechend weniger Signalvalidität für die Funktion Patiens auf als die Wortgestalt [Xen]. Vor dem Hintergrund einer solchen Betrachtung kann das von schwachen Maskulina abstrahierte Patiens-Schema als das prototypische PatiensSchema bestimmt werden.

Beziehen wir nun noch die Überlegungen von Wecker (2016) zu auf paradigmatischen Beziehungen beruhenden Paar-Schemata ein, können noch weitreichendere Konsequenzen zur schemabasierten Modellierung der Agens- und Patiens-Rolle formuliert werden, aus denen dann nicht nur Voraussagen zur unterschiedlichen Verarbeitung von starken und schwachen Maskulina in der

11 Bei Akkusativ-Phrasen schwacher Maskulina wie Junge - den Jungen tritt natürlich alternativ die Gestalt [den $\mathrm{X} n]$ auf. 
Patiens-Rolle, sondern auch für die formal identisch zu den starken Maskulina repräsentierte Agens-Rolle getroffen werden können.

Für unsere Modellierung der schematischen mentalen Repräsentation der Agens- und Patiens-Rolle als Paar-Schemata lassen sich wie in Abbildung 1 dargestellt paradigmatische Verknüpfungen zwischen den Agens- und PatiensSchemata für starke und schwache Maskulina annehmen (angelehnt an Bybees assoziative grammatische Netzwerke). Die Verbindungslinien zwischen dem beispielhaft angeführten starken Maskulinum links (Hund) und dem schwachen Maskulinum rechts (Prinz) symbolisieren die Verknüpfungen zwischen den in den Deklinationsparadigmen formal miteinander übereinstimmenden sprachlichen Elementen, wobei die nicht durchgängigen Linien die Unterschiede zwischen ihnen deutlich machen (Artikelformen, Auslaut/Silbenzahl). Mittig sind die - als kognitive Abstraktionen von den konkreten sprachlichen Einzelexemplaren zu verstehenden - Agens-Patiens-Paar-Schemata dargestellt.

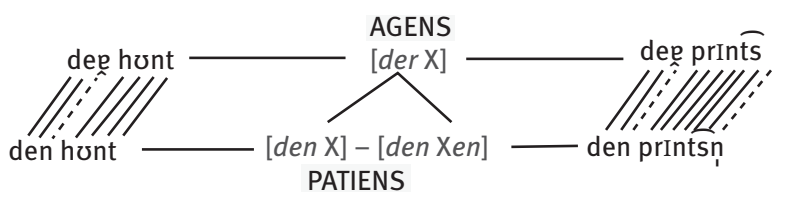

Abb. 1: Agens- und Patiens-Paar-Schemata (Singular).

Durch die Abbildung soll verdeutlicht werden, dass es zwei verschiedene AgensPatiens-Paar-Schemata gibt: einerseits [der X] - [den X] für starke Maskulina, andererseits [der X] - [den Xen] für schwache Maskulina. Den Paar-Schemata kommt für unsere Fragestellung deshalb eine wichtige Bedeutung zu, da sich aus dieser Modellierung nicht allein für das Patiens-Schema der schwachen Maskulina im Vergleich zum Patiens-Schema der starken Maskulina ableiten lässt, dass es bei der Sprachverarbeitung eindeutiger als jenes der starken Maskulina interpretierbar sein sollte, weil es aufgrund seiner Doppelmarkierung in mehrfachem Kontrast zum Agens-Schema steht; darüber hinaus kann im Umkehrschluss auch für das Agens-Schema der schwachen Maskulina angenommen werden, dass es eindeutiger als jenes der starken Maskulina ist, zumal schwache Maskulina im Singular ausschließlich in der Agens-Rolle in der Gestalt [der X] auftreten, während sie in der Patiens-Rolle der Wortgestalt [Xen] entsprechen. Tritt in einer transitiven Singular-Konstruktion also ein schwaches Maskulinum im Slot [der X] auf, sollte es eindeutiger als Agens interpretierbar sein als ein star- 
kes Maskulinum - sofern Sprecherinnen und Sprecher in ihrer mentalen Grammatik (bereits) Agens-Patiens-Paar-Schemata ausgebildet haben. ${ }^{12}$

Bezogen auf unsere morphologietheoretische Fragestellung nehmen wir also an, dass Sprecherinnen und Sprecher im Verlauf ihrer sprachlichen Entwicklung in einem ersten Schritt einfache Agens- und Patiens-Schemata ausbilden. Die Wortgestalt [Xen] wird in diesem Prozess stärker mit der Funktion Patiens als mit der Funktion Agens verknüpft, die Wortgestalt $[\mathrm{X}]$ wird hingegen mit beiden Funktionen assoziiert. In einem zweiten Schritt - mit zunehmender Spracherfahrung - verknüpfen Sprecherinnen und Sprecher die einfachen Schemata zu PaarSchemata. Dadurch assoziieren sie die Wortgestalt [X] im Fall von schwachen Maskulina stärker mit der Funktion Agens, weil sie bei schwachen Maskulina exklusiv in der Agens-Rolle auftritt. Evidenz für morphosyntaktische Schemata und ihre Weiterentwicklung zu Paar-Schemata sollte sich in unserem Experiment also dadurch zeigen, dass die kindlichen Versuchspersonen mit geringerer Spracherfahrung zwar die OVS-Bedingung mit schwachen Maskulina besser verarbeiten als die OVS-Bedingung mit starken Maskulina, sich bei der SVO-Bedingung aber kein Unterschied in Abhängigkeit von der Deklinationsklasse zeigt (einfache Schemata). Für die erwachsenen Versuchspersonen, die über eine höhere Spracherfahrung verfügen und generell eigentlich keine Probleme mit der Identifikation von Agens- und Patiens-Rollen haben sollten, erwarten wir demgegenüber, dass sie (tendenziell) sowohl die SVO- als auch die OVS-Bedingung mit schwachen Maskulina (noch) besser interpretieren können als die SVO- und die OVS-Bedingung mit starken Maskulina (Paar-Schemata).

\section{Hypothesen}

Vor dem Hintergrund der bisher vorliegenden Sprachverarbeitungsstudien und den dargelegten theoretischen Hintergründen lassen sich zusammenfassend folgende Hypothesen ableiten:

12 Auch Ronneberger-Sibold (i.d. B.: 421) nimmt für den Aufbau von Deklinationsklassen die Existenz von Paar-Schemata an, die sie „Wahlverwandtschaften“ nennt, bei denen (einfachen) Grundformschemata die passenden (einfachen) Genitiv-Singular bzw. Nominativ-Pluralformen zugeordnet werden. Ihre mit Kunstwörtertests ermittelten Ergebnisse mit Sprecherinnen und Sprechern verschiedener regionaler Varietäten legen nahe, dass Paar-Schemata Deklinationsklassen konstituieren und von Sprachbenutzerinnen und -benutzern zudem auf einer Prototypikalitätsskala angeordnet werden. 
1) Die morphologische Doppelmarkierung der Patiens-Rolle führt dazu, dass schwache Maskulina besser verarbeitet werden als starke Maskulina, die morphologisch einfach kodiert sind.

2) In Abhängigkeit von der Spracherfahrung der Versuchspersonen werden Unterschiede bei der Verarbeitung von SVO- und OVS-Strukturen mit starken und schwachen Maskulina deutlich, die sich darauf zurückführen lassen, dass sie entweder erst einfache Agens-Patiens-Schemata oder aber bereits Agens-Patiens-Paar-Schemata ausgebildet haben.

\section{Empirische Studie}

\subsection{Probanden, Methode und Testmaterial}

An der Studie nahmen insgesamt 40 Versuchspersonen teil. Neben 14 monolingual deutschsprachigen Erwachsenen, die als Kontrollgruppe mit der höchsten Spracherfahrung fungierten, wurden außerdem 15 monolingual deutschsprachig und elf mehrsprachig aufgewachsene Kinder getestet. Durch diese Probandenauswahl konnten mögliche Unterschiede hinsichtlich der Spracherfahrung, operationalisiert durch die unterschiedlichen Spracherwerbstypen, eruiert werden (Erwachsene $>$ L1-Kinder $>$ L2-Kinder).

Die L1- und L2-Kinder hatten ein Durchschnittsalter von 7;4 Jahren und besuchten die zweite Klasse einer Grundschule in einem Ballungsgebiet NordrheinWestfalens. Die mehrsprachigen Kinder waren nach Auskunft der Lehrkräfte alle in Deutschland geboren, hatten aber erst seit dem Besuch des Kindergartens im Alter von drei bis vier Jahren regelmäßigen Kontakt mit dem Deutschen, sodass es sich bei ihnen um frühe, sukzessive L2-Lernende handelt. Die L1 der mehrsprachigen Kinder ist Türkisch.

Von den Kindern wurde der allgemeine Sprachstand im Deutschen anhand einer Elicited-Imitation-Aufgabe (Erlam 2006) ermittelt. Dabei wurden die Kinder gebeten, 24 Sätze zu wiederholen, von denen jeweils die Hälfte grammatikalisch richtig bzw. falsch waren. Die Fehler in den ungrammatischen Testsätzen betrafen die Bereiche Kasusmarkierung, Adjektivflexion, Subjekt-Verb-Kongruenz und Wortstellung. Bei der Auswertung wurden Satzveränderungen beim Wiederholen korrekt vorgegebener Sätze sowie Korrekturen bei den ungrammatischen Sätzen analysiert. Die L2-Kinder erzielten im Durchschnitt signifikant schlechtere Ergebnisse als die L1-Kinder. Die untersuchten L1- und L2-Kinder unterscheiden sich also hinsichtlich ihres Sprachstandes im Deutschen voneinander, sodass auch bezogen auf die Verarbeitung semantischer Rollen Unterschiede zwischen den beiden Gruppen zu erwarten sind. 
Bei der Untersuchung handelte es sich um eine Eye Tracking Studie (Visual World Paradigm). In solchen Experimenten verarbeiten die Versuchspersonen parallel auditive (sprachlicher Input) und visuelle Stimuli (Bildmaterial); letztere werden ihnen auf einem Bildschirm präsentiert. Der sprachlich zu verarbeitende Input steht in Beziehung zum visuell präsentierten Bildmaterial. Während der Sprachverarbeitung werden die Blickbewegungen und -fixationen der Versuchspersonen aufgezeichnet, um zu erfassen, ob und wann sie ihre Blicke auf welche visuellen Stimuli richten. Es wird angenommen, dass die Blickbewegungen bzw. -fixationen der Versuchspersonen während der Verarbeitung des auditiven Inputs Aufschluss über die sprachliche Verarbeitung in Echtzeit geben (vgl. z. B. Berends, Bouwer \& Sprenger 2015).

In unserem Experiment bestand das Testmaterial aus 16 experimentellen WFragen mit einem zweifaktoriellen Untersuchungsdesign, das zu zwei experimentellen Listen führte. Der erste Faktor war „Wortstellung“ mit den zwei Stufen „subjekt-initial“ (SVO-Fragen) und „objekt-initial“ (OVS-Fragen), sodass acht der insgesamt $16 \mathrm{~W}$-Fragen mit einem Subjekt und acht mit einem Objekt begannen.

Der zweite Faktor war „Morphologie“ mit den zwei Stufen „schwaches Maskulinum“ und „starkes Maskulinum“, sodass jeweils die Hälfte der SVO- und OVS-Fragen ein starkes Maskulinum und die andere Hälfte ein schwaches Maskulinum in der ersten NP enthielt.

Alle experimentellen Fragen begannen mit dem Interrogativartikel welch-, entweder im Nominativ (welcher) oder Akkusativ (welchen), gefolgt von einem starken bzw. schwachen Maskulinum. Tabelle 4 stellt die vier unterschiedlichen Bedingungen exemplarisch dar.

Tab. 4: Darstellung der vier W-Fragetypen.

\begin{tabular}{|c|c|c|c|c|}
\hline \multirow{2}{*}{$\frac{\text { Morphologie/Wortstellung }}{\text { Starkes Maskulinum }}$} & \multicolumn{2}{|c|}{ SVO-Fragen } & \multicolumn{2}{|c|}{ OVS-Fragen } \\
\hline & a) & $\begin{array}{l}\text { Welcher Arztø } \\
\text { beobachtet die kleine } \\
\text { Frau? }\end{array}$ & b) & $\begin{array}{l}\text { Welchen Arztø } \\
\text { beobachtet die kleine } \\
\text { Frau? }\end{array}$ \\
\hline Schwaches Maskulinum & c) & $\begin{array}{l}\text { Welcher Prinz } \varnothing \\
\text { beobachtet die kleine Frau? }\end{array}$ & d) & $\begin{array}{l}\text { Welchen Prinzen } \\
\text { beobachtet die kleine Frau? }\end{array}$ \\
\hline
\end{tabular}

Nach der ersten NP folgte ein finites Verb im Präsens. Dafür wählten wir die zwei lexikalischen Verben beobachten und küssen, die Handlungen zwischen zwei belebten Akteuren ausdrücken.

Die zweite NP war aus einem femininen Substantiv und einem ihm vorangehenden attributiven Adjektiv zusammengesetzt. Die femininen Substantive 
wurden wie die Verben eingeschränkt variiert, um eine Beeinflussung der Satzverarbeitung durch eine lexikalische Alternation auszuschließen. Wir nutzten insgesamt vier Feminina: in Kombination mit Maskulina, die menschliche Akteure bezeichnen, Oma und Frau, in Kombination mit Maskulina, die tierische Akteure bezeichnen, Gans und Kuh.

Die Akteurpaare wurden so ausgewählt, dass für keinen Akteur eine stärkere Agentivität ausgemacht werden konnte. Sowohl semantische als auch prosodische Faktoren, die die Interpretation der Sätze hätten beeinflussen können (vgl. Abschnitt 2), wurden neutralisiert. D. h. zum einen, dass alle Substantive das Merkmal [+belebt] aufwiesen und zum anderen, dass die Substantive, ob Subjekt oder Objekt, in keiner Satzposition intonatorisch hervorgehoben wurden. Während objekt-initiale Deklarativsätze eine spezielle Informationsstruktur des Kontextes voraussetzen, die die aus pragmatischer Perspektive als markiert zu bezeichnende Objekttopikalisierung nachvollziehbar machen (Musan 2010), sind objekt-initiale W-Fragen nicht markierter als subjekt-initiale W-Fragen (Welcher Arzt beobachtet die Frau? vs. Welchen Arzt beobachtet die Frau?). Entsprechend haben wir uns für den Einsatz von W-Fragen und gegen die Verwendung von Deklarativsätzen entschieden. ${ }^{13}$

Die visuellen Stimuli bestanden aus Bildern, auf denen jeweils drei Referenten dargestellt wurden, die entweder die Handlung ,küssen“ oder ,beobachten“ ausführten. Die zwei äußeren Akteure waren jeweils identisch, bezogen sich auf das maskuline Substantiv der W-Frage und konnten je nach Interpretation entweder als Agens bzw. als Patiens dekodiert werden. In der Mitte des Bildes wurde das in der W-Frage enthaltene feminine Substantiv dargestellt, vgl. Abbildung 2.

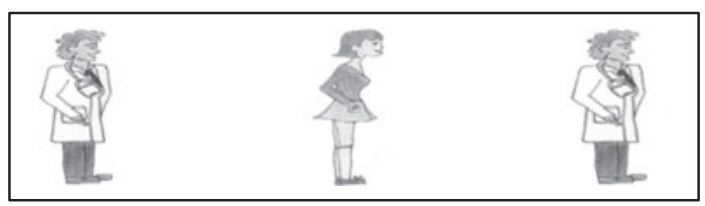

Abb. 2: Bild zu den W-Fragen mit dem Verb ,beobachten“, im Original und Experiment farbig dargestellt.

13 An vergleichbarem Testmaterial mit Deklarativsätzen und prosodisch neutralisierten, d. h. intonatorisch nicht betonten satzinitialen Objekten, wird häufig kritisiert, dass solche Sätze unnatürlich seien und die Ergebnisse solcher Experimente entsprechend keinen Aufschluss über die natürliche Sprachverarbeitung gäben. 
Bei der Durchführung des Experiments hörten die Versuchspersonen vor den experimentellen W-Fragen zwei Warm-up Items. Die Präsentationsreihenfolge der experimentellen Items wurde zwischen den Versuchspersonen randomisiert und durch insgesamt zehn Filler-Items unterbrochen. Nach jedem Trial erhielten die kindlichen Versuchspersonen ein kindgerechtes Belohnungsbild.

Die ausgewählten acht schwachen und acht starken Maskulina waren jeweils zur einen Hälfte Tier-, zur anderen Hälfte Personenbezeichnungen. Die Substantive wurden außerdem nach ihrer Silbenzahl nivelliert, wobei jeweils eine Hälfte einsilbig, die andere Hälfte zweisilbig war, vgl. erneut Tabelle 3 in Abschnitt 3.

\subsection{Durchführung, Kodierung und statistische Auswertung}

Das Experiment wurde im Anschluss an die Elicited-Imitation-Aufgabe mit jeder Versuchsperson einzeln auf einem Laptop durchgeführt. Vor der Präsentation der experimentellen W-Fragen wurden die Versuchspersonen gebeten, alle auf den visuellen Stimuli abgebildeten Akteure zu benennen, um eine Beeinflussung der Verarbeitung durch nicht bekannte Substantive ausschließen zu können. Waren die Substantive den Versuchspersonen nicht bekannt, wurden sie in diesem Testteil durch die Bilder eingeführt (Familiarisierung).

Die Versuchspersonen saßen mit einem Abstand von ca. 50 bis $80 \mathrm{~cm}$ vor einem 21-Zoll-Bildschirm und erhielten die Instruktion, die auf dem Bildschirm erscheinenden Bilder anzuschauen, während die zuvor von uns aufgenommenen W-Fragen als auditiver Input vorgespielt wurden. Die Tondateien wurden von einem männlichen L1-Sprecher eingesprochen und digital aufgenommen, der auditive Input war also für alle Versuchspersonen identisch.

Während der Satzverarbeitung wurden die Blickbewegungen der Kinder und Erwachsenen mittels eines Tobii X1 Light Eye Trackers erfasst (Online-Aufgabe). Die Bilder wurden den Versuchspersonen zunächst 2.500 Millisekunden lang ohne auditiven Stimulus präsentiert. Dies erlaubte den Versuchspersonen, den visuellen Input vor dem Einsetzen des sprachlichen Inputs zu verarbeiten.

Für die Offline-Auswertung wurden die Antworten der Versuchspersonen auf die Fragen durch Mouse-Klicks erfasst. Dazu wurden sie gebeten, nach der Verarbeitung der W-Fragen durch einen Mouse-Klick die Person / das Tier auszuwählen, auf die/das sich die Frage bezog. Dabei wurde betont, dass es erforderlich sei, den aufgenommenen Satz bis zum Ende zu hören und erst dann eine Auswahl zu treffen. Bei der statistischen Auswertung wurden Klicks auf den richtigen Referenten ein Punkt, Klicks auf den falschen Referenten oder die Referentin in der Mitte keine Punkte zugewiesen. 
Für die Online-Auswertung wurde gemessen, in welcher Häufigkeit die Versuchspersonen die unterschiedlichen Referenten auf den Bildern während des Hörens bestimmter Satzsegmente mit ihren Blicken fixieren. Dafür wurde der gesamte Satz in drei Zeitfenster unterteilt (Tabelle 5).

Tab. 5: Zeitfenster.

\begin{tabular}{|c|c|c|}
\hline & Zeitfenster 1 & Zeitfenster 2-1 \\
\hline & $\begin{array}{l}\text { Anfang des Satzes bis zum } \\
\text { Onset des finiten Verbs }\end{array}$ & $\begin{array}{l}\text { Onset des finiten Verbs } \\
\text { bis zum Ende des Satzes }\end{array}$ \\
\hline a) SVO stark & Welcher Arztø & \multirow{4}{*}{ beobachtet die kleine Frau? } \\
\hline b) SVO schwach & Welcher Prinz & \\
\hline c) OVS stark & Welchen ArztØ & \\
\hline d) OVS schwach & Welchen Prinzen & \\
\hline
\end{tabular}

Während des ersten Zeitfensters hörten die Versuchspersonen den Interrogativartikel und das erste Substantiv (schwaches bzw. starkes Maskulinum). In diesem Zeitfenster können die W-Fragen aufgrund der Kasusmarkierungen am Interrogativartikel bzw. ggf. durch die zusätzliche Markierung an den schwachen Maskulina selbst bereits als SVO- oder OVS-Strukturen disambiguiert werden. Die Dauer des ersten Zeitfensters wurde jeweils so berechnet, dass sie bei jedem Item zum Onset des finiten Verbs endete.

Während des zweiten Zeitfensters hörten die Versuchspersonen das finite Verb und die zweite expandierte NP (Artikelwort, attributives Adjektiv, feminines Substantiv). Aufgrund seiner im Vergleich zum ersten Zeitfenster längeren Dauer wurde es in zwei gleichlangen Teilen analysiert. Die Gesamtdauer des zweiten Zeitfensters erstreckte sich vom Onset des finiten Verbs bis zum Satzende.

Die statistische Auswertung der Blickbewegungen und der Antwortkorrektheit erfolgte mittels linear gemischter Regressionsmodelle bzw. gemischter logistischer Regressionsmodelle und wurde mithilfe des lme4-Pakets (Bates, Mächler, Bolker \& Walker 2015) und languageR (Baayen 2008) der Software R durchgeführt. Effekte, die für unsere Forschungsfrage von Interesse sind, sind Haupteffekte von „Wortstellung“ (SVO- versus OVS-Fragen), „Morphologie“ (starkes vs. schwaches Maskulinum) sowie „Versuchsgruppe“ (L1-Erwachsene, L1-Kinder, L2-Kinder) oder Interaktionen zwischen diesen Faktoren.

Analysiert wurden die Blickbewegungen der drei Gruppen während des Hörens aller W-Fragen, unabhängig davon, ob sie die Fragen offline korrekt oder nicht korrekt interpretierten. Somit wollten wir herausfinden, wie die Versuchspersonen die morphosyntaktischen Hinweisreize online wahrnehmen, auch 
wenn sie bei der finalen Offline-Aufgabe bzw. der Auswahl des richtigen Referenten Schwierigkeiten hatten.

\subsection{Ergebnisse}

\subsubsection{Offline-Ergebnisse}

Tabelle 6 zeigt die Antwortkorrektheit der drei Vergleichsgruppen in den vier Bedingungen. Die Erwachsenen zeigten in keiner der vier Bedingungen Schwierigkeiten bei der Auswahl des richtigen Referenten. In der inferenzstatistischen Analyse wurden keine signifikanten Effekte gefunden. Die Kindergruppen schnitten hingegen in den SVO-Bedingungen insgesamt deutlich besser ab als in den OVS-Bedingungen, was durch den Haupteffekt des Faktors „Wortstellung“ in beiden Analysen bestätigt wird (L1-Kinder: Estimate $=-2.2725$, Std. $=0.3220, z=-7.058$, $\mathrm{p}=$ 0; L2-Kinder: Estimate $=-3.0880$, Std. $=0.5984, \mathrm{z}=5.161, \mathrm{p}=0$ ).

Tab. 6: Antwortkorrektheit der drei Gruppen (in \%).

\begin{tabular}{lrrrr}
\hline$\%$ & SVOstark & SVOschwach & OVSstark & OVSschwach \\
\hline L1-Erwachsene & 98 & 95 & 95 & 100 \\
L1-Kinder & 95 & 92 & 34 & 41 \\
L2-Kinder & 98 & 98 & 23 & 36 \\
\hline
\end{tabular}

Bezogen auf den Faktor „Morphologie“ ist nur deskriptiv festzustellen, dass die OVS-Bedingungen mit schwachen Maskulina bei allen drei Vergleichsgruppen zu einer höheren Antwortkorrektheit führte als mit starken Maskulina.

\subsubsection{Online-Ergebnisse}

Um eine Gesamtübersicht zum Blickbewegungsverlauf der drei Gruppen zu gewinnen, werden in Abbildung 3 die Blickbewegungen auf den zum Bild passenden Referenten dargestellt, die vom Anfang bis zum Ende der Verarbeitungszeit der W-Fragen aufgezeichnet wurden. Dabei fassen wir die Blickbewegungen aller Versuchspersonen einer Vergleichsgruppe für jedes Zeitfenster im Durchschnitt zusammen und stellen diese Durchschnittswerte, jeweils differenziert für die drei unterschiedlichen Vergleichsgruppen und Bedingungen, in Säulendiagrammen einander gegenüber. 
Die drei Teilabbildungen zeigen somit die Prozentanteile der Blickbewegungen auf den korrekten Referenten (im Vergleich zu den Blicken auf die anderen beiden Referenten). Jede Abbildung bezieht sich auf ein Zeitfenster im Satz, in dem die Versuchspersonen die unter den Abbildungen bezeichneten Segmente der W-Fragen hörten. Liegen die Werte bei 30\%, können zufällige Fixationen nicht ausgeschlossen werden. Liegen die Werte über 30\%, handelt es sich um Blicke auf den richtigen Referenten.

Als allgemeines Ergebnis kann zunächst festgehalten werden, dass alle drei Testgruppen in allen vier Bedingungen die visuellen Stimuli sowohl über als auch unter dem Zufallswert von 30\% fixieren. Daraus lässt sich schließen, dass alle Vergleichsgruppen semantische Rollen inkrementell zuweisen können, also auch die L1- und L2-Kinder im Alter von sieben Jahren.

Durch die inferenzstatistische Analyse konnten keine signifikanten Effekte festgestellt werden. Wir beschränken uns deshalb auf eine deskriptive Beschreibung der Ergebnisse, die entsprechend nur als Tendenzen interpretiert werden dürfen.

\section{Wortstellung}

Für die erwachsenen L1-Versuchspersonen kann auch für die Online-Ergebnisse (wie schon für die Offline-Ergebnisse) konstatiert werden, dass sie SVO- und OVS-Fragen gleich gut verarbeiten. Das erste Zeitfenster und der erste Teil des zweiten Zeitfensters zeigen zwar einen Unterschied zwischen den beiden Bedingungen, der verdeutlicht, dass die Verarbeitung von objekt-initialen Fragen zeitlich versetzt zu den SVO-Fragen erfolgt und die Erwachsenen somit auch bei der Dekodierung der OVS-Strukturen zunächst der agent first-Strategie folgen; im zweiten Teil des zweiten Zeitfensters schauen die Erwachsenen aber in der gleichen Häufigkeit wie in der SVO-Bedingung auf den richtigen Referenten, was bedeutet, dass sie aufgrund der erfolgreichen Verarbeitung morphologischer Hinweise im Verlauf des Verarbeitungsprozesses ihre agent first-Strategie revidieren.

Im Vergleich dazu zeigt sich für beide kindlichen Probandengruppen - ebenfalls übereinstimmend mit den Befunden unserer Offline-Ergebnisse sowie mit den Ergebnissen der in Abschnitt 2 dargestellten Studien -, dass die SVO-Bedingung deutlich erfolgreicher als die OVS-Bedingung verarbeitet wird. Beide Gruppen verfolgen auch bei OVS-Strukturen überwiegend die agent first-Strategie, nur ein kleiner Teil der Kinder scheint im Verlauf des Verarbeitungsprozesses auf die morphologischen Hinweise, die die Identifikation der satzinitialen NP als Patiens ermöglicht, zu reagieren. 

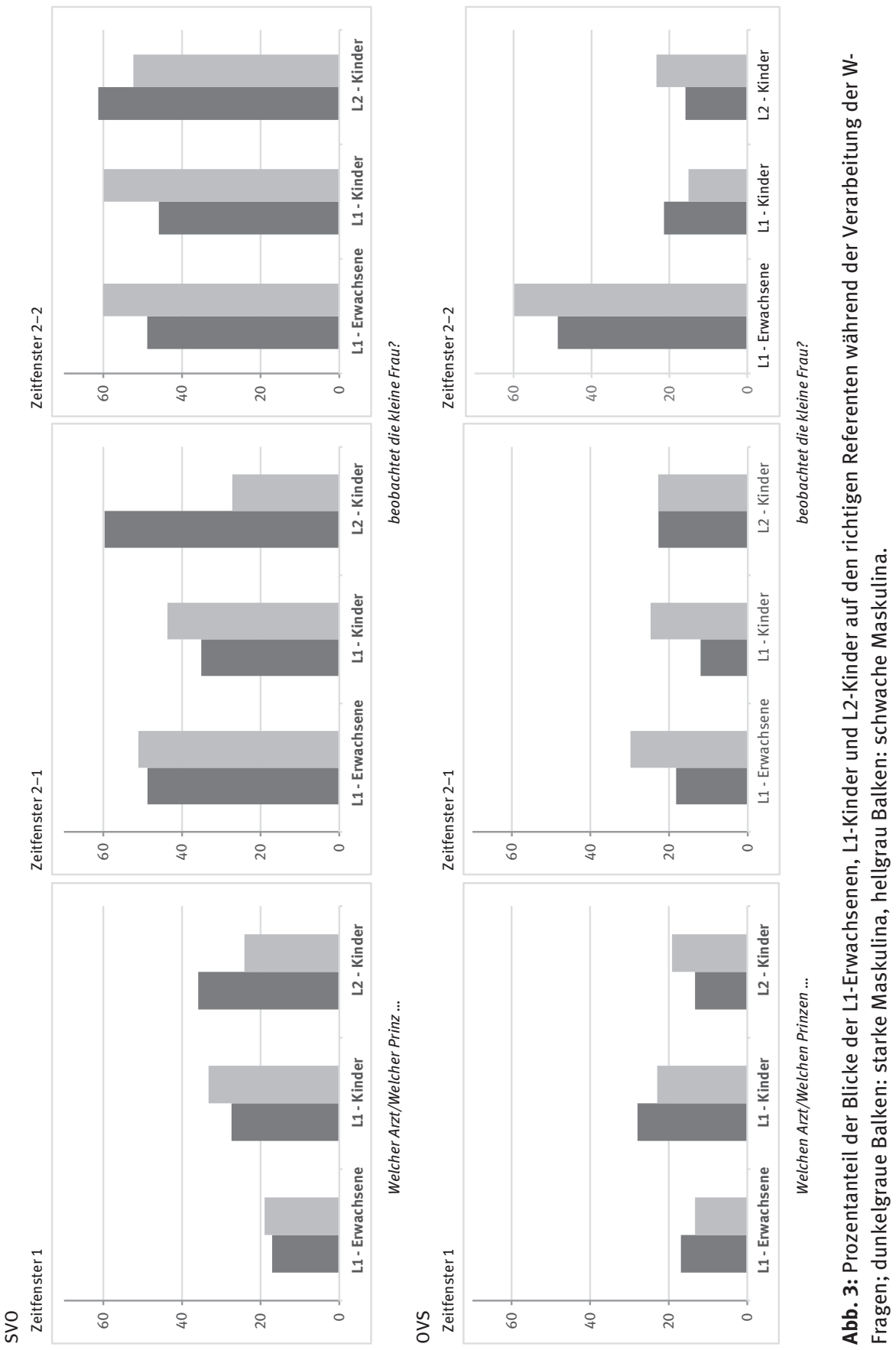


\section{Morphologie}

Im Vergleich der Verarbeitungsdaten von starken und schwachen Maskulina zeigt sich für die erwachsenen L1-Versuchspersonen, dass sie SVO- und OVSFragen mit schwachen Maskulina insgesamt erfolgreicher verarbeiten als Fragen mit starken Maskulina. Für beide Bedingungen wird zudem deutlich, dass die Blicke der Erwachsenen auf den richtigen Referenten im Verarbeitungsverlauf vom ersten Zeitfenster bis zum zweiten Teil des zweiten Zeitfensters zunehmen, wenn die W-Frage ein schwaches Maskulinum enthält.

Für die L1-Kinder trifft dieser Befund nur auf die SVO-Fragen zu: Bei der Verarbeitung von subjekt-initialen Fragen mit schwachen Maskulina schauen sie im Verlauf zunehmend und insgesamt häufiger auf den richtigen Referenten als bei SVO-Fragen mit starken Maskulina. Bei OVS-Fragen zeigt sich nur im ersten Teil des zweiten Zeitfensters eine bessere Verarbeitung der NPs mit morphologischer Doppelmarkierung.

Die L2-Kinder zeigen den L1-Kindern gegenüber ein konträres Verarbeitungsmuster: SVO-Fragen mit starken Maskulina verarbeiten sie im gesamten Verlauf erfolgreicher als SVO-Fragen mit schwachen Maskulina. Für die OVSFragen zeigt sich wiederum wie bei den L1-Erwachsenen, dass sie häufiger auf den richtigen Referenten schauen, wenn das Objekt eine Doppelmarkierung aufweist. Die Blicke auf den richtigen Referenten steigen im Verarbeitungsverlauf leicht an (von 19\% auf 23\%).

\section{Diskussion}

Zur Diskussion der Ergebnisse greifen wir die in Abschnitt 5 formulierten Fragestellungen und Hypothesen wieder auf, wobei wir an dieser Stelle noch einmal darauf hinweisen, dass unsere Dateninterpretation vor dem Hintergrund der inferenzstatistisch größtenteils nicht signifikanten Ergebnisse entsprechend vorsichtig formuliert werden muss.

1) Führt eine doppelte morphologische Kennzeichnung der Patiens-Rolle in OVS-Strukturen zur besseren Verarbeitung nicht-agentiver Rollen als die einfach kodierte Patiens-Rolle?

Da verschiedene Studien zur Verarbeitung von objekt-initialen (Deklarativoder Frage-)Sätzen gezeigt haben, dass Vor- und Grundschulkinder mit Deutsch als L1 oder L2 morphologische Hinweise zur Dekodierung von OVS-Strukturen im Vergleich zu Erwachsenen kaum nutzen (Schipke et al. 2012; Roesch \& Chondrogianni 2014, 2015, 2016; Fengler, Meyer \& Friederici 2015; Cristante 2016; 
Gamper 2016; Cristante \& Schimke 2018; Schouwenaars, Hendriks \& Ruigendijk 2018), haben wir danach gefragt, ob eine doppelte morphologische Kennzeichnung der Patiens-Rolle die Wahrnehmung morphologischer Hinweise und damit die (zielsprachliche) Verarbeitung solcher Strukturen befördert. In unserem Eye Tracking Experiment alternieren zur Überprüfung dieser Frage starke und schwache Maskulina in der objekt-initialen Position (am Beispiel von W-Fragen). Die schwachen Maskulina weisen in der Patiens-Rolle eine doppelte morphologische Markierung auf, am Artikelwort und am Substantiv selbst, während starke Maskulina nur am Artikelwort als Patiens gekennzeichnet werden.

Unsere Offline-Ergebnisse bestätigen (nur) deskriptiv für alle drei von uns getesteten Probandengruppen (L1-Erwachsene, L1-Kinder, L2-Kinder) unsere Annahme, dass doppelt markierte Patiens-Rollen in OVS-Fragen häufiger zur richtigen Referentenauswahl führen als ausschließlich durch den Interrogativartikel gekennzeichnete Patiens-Rollen. Das Ausmaß, in dem das geschieht, ist abhängig von der Spracherfahrung der Versuchspersonen und spiegelt sich in der festgestellten Reihenfolge der Probandengruppen: Die Erwachsenen verarbeiten den doppelten morphologischen Hinweis sehr erfolgreich $(100 \%)$, gefolgt von den L1-Kindern (41\%), die wiederum vor den L2-Kindern (36\%) rangieren. Die Online-Daten stimmen mit diesem Befund weitgehend überein. So trifft auf deskriptiver Ebene für alle zu, dass sie die Doppelmarkierung im Verarbeitungsverlauf erfolgreich interpretieren und dadurch die von ihnen zunächst verfolgte agent first-Strategie revidieren können, wobei die Erwachsenen - gemessen am Endpunkt des Verarbeitungsverlaufs - deutlich häufiger als die L2-Kinder auf die Doppelmarkierung reagieren (60\% vs. 23\%). Die L1-Kinder schauen zu diesem Zeitpunkt zwar nur zu 15\% auf die richtigen Referenten, weisen dafür aber bereits im ersten Abschnitt des zweiten Zeitfensters eine Fixationsquote auf den richtigen Referenten von 25\% auf.

Im Vergleich der Online- und Offline-Ergebnisse aller drei Gruppen kann unsere Annahme deskriptiv demnach eher als bestätigte denn als widerlegte Tendenz angesehen werden: Doppelmarkierungen in OVS-Strukturen unterstützen die Verarbeitung von satzinitialen Patiens-Rollen, sofern morphologische Hinweise zur Disambiguierung semantischer Rollen erkannt werden (L1-Erwachsene vs. L1- und L2-Kinder).

Als inkonsistent bleibt aber das Online-Ergebnis der L1-Kinder festzuhalten. Aufgrund ihrer höheren Spracherfahrung gegenüber den L2-Kindern und ihres Offline-Ergebnisses wäre anzunehmen gewesen, dass sie die Doppelmarkierung auch online besser verarbeiten als die L2-Kinder. Zwei Überlegungen sollen hierzu angeführt werden: Zum einen zeigte sich, wie bereits angemerkt, bei den 
L1-Kindern ja schon im Verarbeitungsverlauf im ersten Teil des zweiten Zeitfensters, dass die Doppelmarkierung gegenüber der einfachen Markierung häufiger als morphologischer Hinweis erkannt und verarbeitet wird. Zum anderen kann bei den L2-Kindern online der Einfluss einer L1-Verarbeitungsstrategie vermutet werden. In ihrer flektierend-agglutinierenden L1 Türkisch werden Form-Funktions-Relationen durch morphologische Mittel sehr transparent markiert (Bassarak \& Jendraschek 2004; Göksel \& Kerslake 2011), so dass sie für morphologische Markierungen besonders sensitiv sein dürften. Auch bei der Satzverarbeitung in der L2 Deutsch könnten sie demnach unbewusst bereits auf die morphologischen Doppelmarkierungen reagieren, wobei diese Verarbeitungsstrategie in der Offline-Entscheidung aber noch keinen (durchgängigen) Niederschlag findet.

2) Werden in Abhängigkeit von der Spracherfahrung der Versuchspersonen Unterschiede bei der Verarbeitung von SVO- und OVS-Strukturen mit starken und schwachen Maskulina deutlich, die sich darauf zurückführen lassen, dass entweder erst einfache Agens-Patiens-Schemata oder aber bereits Agens-Patiens-Paar-Schemata ausgebildet wurden?

Interpretieren wir die Offline- und Online-Daten unserer erwachsenen L1-Sprecherinnen und Sprecher vor dem Hintergrund gebrauchsbasierter Schema-Modelle zur mentalen Repräsentation sprachlichen Wissens (Bybee 1985, 1988, 2010; Köpcke 1993; Wecker 2016), bieten sie (wenn auch nur) deskriptiv Evidenz dafür, das Vorhandensein entsprechender morphosyntaktischer Paar-Schemata als Muster der mentalen Repräsentation anzunehmen. So zeigt sich offline und online für die OVS-Bedingung, dass die doppelt markierten Patiens-Rollen der schwachen Maskulina besser verarbeitet werden als die einfach markierten Patiens-Rollen der starken Maskulina; dieser Befund lässt sich online auch für die SVO-Bedingung feststellen: Obwohl sich die Agens-Rolle in ihrem formalen Ausdruck zwischen schwachen und starken Maskulina nicht unterscheidet ([der X]), schauen die erwachsenen Versuchspersonen bei den W-Fragen mit schwachen Maskulina häufiger auf die richtigen Referenten als bei den W-Fragen mit starken Maskulina. Diese Beobachtung lässt sich vor dem Hintergrund des Schema-Ansatzes dadurch erklären, dass die erwachsenen L1-Sprecherinnen und Sprecher Agens-Patiens-Paar-Schemata für beide Deklinationsklassen ausgebildet haben: Ein schwaches Maskulinum, das nicht dem Schema [Xen] entspricht, kann auf der Basis der bloßen Wortgestalt eindeutiger als ein Agens interpretiert werden als ein starkes Maskulinum, weil für ein nicht auf -en auslautendes schwaches Maskulinum in einem transitiven Satz keine andere Interpretation in Frage kommt. Demgegenüber sind von der Wortgestalt der starken Maskulina im NOM 
SG bzw. AKK SG keine unmittelbaren Rückschlüsse auf die Rollenfunktion möglich, woraus sich der Unterschied bei der Verarbeitung dieser beiden Strukturen ergibt.

Die Online-Daten der Vergleichsgruppe mit der geringsten Spracherfahrung im Deutschen, der kindlichen L2-Versuchspersonen, bieten konsistent dazu Hinweise auf den Ausgangspunkt der Herausbildung solcher Agens- und Patiens-Schemata. Diese Daten können so interpretiert werden, dass sich die L2-Lernenden noch im Aufbauprozess einfacher Agens- und Patiens-Schemata befinden, wobei sich das Erkennen und Nutzen der morphologischen Hinweise (zunächst) durch die doppelt markierten NPs initiiert zu vollziehen scheint. Dafür sprechen sowohl die Offline- als auch die Online-Daten, zumal objekt-initiale W-Fragen mit schwachen Maskulina jeweils häufiger richtig verarbeitet werden als objekt-initiale W-Fragen mit starken Maskulina. Dass sie in der SVO-Bedingung die schwachen Maskulina nicht bevorzugter verarbeiten als die starken Maskulina, verweist darauf, dass die L2-Lernenden eben noch keine Paar-Schemata ausgebildet haben und einfache Agens- bzw. Patiens-Schemata in ihrem assoziativen grammatischen Netzwerk noch nicht miteinander verknüpft haben.

Die Daten der L1-Kinder fügen sich in den hier modellierten Erwerbsverlauf, bei dem die L2-Kinder den Ausgangs-, die Erwachsenen den Endpunkt des Erwerbsprozesses markieren, ein: So zeigt sich in den Online-Daten der SVO-Bedingung wie bei den Erwachsenen ein besseres Ergebnis für die Verarbeitung von schwachen Maskulina. Diesen Befund können wir wie bei den Erwachsenen in Kombination mit den Ergebnissen zur OVS-Bedingung als Hinweis auf das Vorhandensein von Agens-Patiens-Paarschemata werten. Diese Interpretation ist für die Daten der L1-Kinder auch mit der Beobachtung kompatibel, dass sich bei ihnen in der OVS-Bedingung mit schwachen Maskulina ein besseres Ergebnis ein solches wird insgesamt aus den Offline-Daten der L1-Kinder ersichtlich - nur im ersten Teil des zweiten Zeitfensters zeigt. Das Ansteigen der Fixationsfrequenz bei den starken Maskulina am Endpunkt des Verarbeitungsverlaufs lässt sich mit der (beginnenden) Etablierung des Agens-Patiens-Paarschemas für die starken Maskulina erklären - ein Prozess, der bei den L2-Kindern noch nicht begonnen hat und bei den Erwachsenen bereits abgeschlossen ist.

\section{Schlussbetrachtung und Ausblick}

Unsere Untersuchung zur Verarbeitung von morphologisch einfach und doppelt markierten Patiens-Rollen in objekt-initialen transitiven Strukturen des 
Deutschen zeigt weder für erwachsene (hier wohl nicht überraschend) noch für kindliche L1- und L2-Sprecherinnen und -Sprecher des Deutschen inferenzstatistisch signifikante Effekte hinsichtlich einer besseren Verarbeitung von morphologisch doppelt gekennzeichneten Patiens-Rollen. Für die kindlichen L1- und L2-Sprecherinnen und -Sprecher stimmen damit unsere Untersuchungsergebnisse mit den Befunden jüngerer Satzverarbeitungsstudien mit kindlichen L1- und L2-Lernenden des Deutschen insofern überein, als dass morphologische Hinweise zur Dekodierung semantischer Rollen bis zu einem Alter von ca. sieben Jahren generell noch kaum genutzt werden - also auch dann nicht, wenn die morphologischen Hinweise verstärkt werden.

Die deskriptiven Ergebnisse, die den von uns erwarteten Unterschied zugunsten einer besseren Interpretierbarkeit einer doppelt markierten Patiens-Rolle tendenziell zeigen, können im Rahmen von gebrauchsbasierten Schema-Ansätzen jedoch schlüssig interpretiert werden. So konnten wir vor diesem theoretischen Hintergrund die deskriptiven Unterschiede zwischen den drei Untersuchungsgruppen so erklären, dass Sprecherinnen und Sprecher im Verlauf ihrer sprachlichen Entwicklung zunächst einfache Agensund Patiens-Schemata ausbilden, die sie mit zunehmender Spracherfahrung zu Paar-Schemata weiterentwickeln, die auf paradigmatischen Verknüpfungen zwischen Wortformen beruhen (vgl. Ronneberger-Sibold i.d.B. und aus funktional-strukturalistischem Blickwinkel Bittner i.d. B.).

Dass der Unterschied bei der Verarbeitung beider Bedingungen (starke vs. schwache Maskulina) bei den erwachsenen Versuchspersonen nur deskriptiv festzustellen ist, kann darauf zurückgeführt werden, dass sie morphologische Hinweise zur Satzinterpretation ohnehin nutzen, wie die hohen Werte zur richtigen Verarbeitung einfach markierter Patiens-Rollen veranschaulichen. Insofern sind für diese Untersuchungsgruppe die (wenn auch minimalen) Überlegenheitstendenzen der schwachen Maskulina bei der Verarbeitung sowohl der OVS- als auch der SVO-Bedingung allemal aufschlussreich.

Um die durch unser Experiment festgestellten Tendenzen verifizieren $\mathrm{zu}$ können, wäre es für zukünftige Untersuchungen erforderlich, die Anzahl der Versuchspersonen zu erhöhen. Weiterhin wäre anzustreben, individuelle Verarbeitungsstrategien zu berücksichtigen. So könnte es bezogen auf Erkenntnisse zur sprachlichen Entwicklung aufschlussreich sein, die Online-Daten nur von denjenigen kindlichen Versuchspersonen zu analysieren, für die sich durch die Offline-Entscheidungen zeigt, dass sie zur Disambiguierung semantischer Rollen morphologische Hinweise berücksichtigen. Interessant könnte auch sein, bei der Auswertung der schwachen Maskulina zu berücksichtigen, ob die entweder additiv silbische (-en) oder silbenschließende (-n) Kennzeichnung der Pa- 
tiens-Rolle Einfluss auf die Verarbeitung ausübt. Mit dem Blick auf den Befund von Indefrey (2002), dass sich der produktive Erwerb des schwachen Flexionsmusters im Alter zwischen fünf und acht Jahren vollzieht, ist die Untersuchung von Verarbeitungsstrategien von Kindern jenseits eines Alters von sieben Jahren naheliegend.

\section{Literatur}

Ágel, Vilmos \& Dagobert Höllein (i.d. B.): Satzbaupläne als Zeichen: die semantischen Rollen des Deutschen in Theorie und Praxis.

Bassarak, Armin \& Geert Jendraschek (2004): Türkisch. In Geert Booij, Christian Lehmann \& Joachim Mugdan (Hrsg.), Morphologie. Ein internationales Handbuch zur Flexion und Wortbildung. Bd. 2, 1358-1366. Berlin: De Gruyter.

Bates, Elisabeth \& Brian MacWhinney (1989): Functionalism and the Competition Model. In Brian MacWhinney \& Elisabeth Bates (Hrsg.), The Crosslinguistic Study of Language Processing, 3-73. New York: Cambridge University Press.

Bates, Douglas, Mächler Martin, Bolker Ben, \& Walker, Steve (2015): Fitting Linear MixedEffects Models Using Ime4. Journal of Statistical Software 67(1).

Baayen, R. Harald (2008): Analyzing linguistic data: A practical introduction to statistics using $R$. Cambridge: Cambridge University Press.

Berends, Sanne M., Susanne M. Brouwer \& Simone A. Sprenger (2015): Eye tracking and the visual world paradigm. In Monika S. Schmid, Sanne M. Berends, Christopher Bergmann, Susanne M. Brouwer, Nienke Meulman, Bregtje J., Seton, Simone A. Sprenger \& Laurie A. Stowe (Hrsg.), Designing Research on Bilingual Development: Behavioural and Neurolinguistic Experiments, 55-80. Cham: Springer.

Bever, Thomas G. (1970): The cognitive basis for linguistic structures. In John R. Hayes (Hrsg.), Cognition and the development of language, 279-362. New York: Wiley.

Bittner, Dagmar (i. d. B.): Strukturalistische vs. gebrauchsbasierte Modellierung des Erwerbs der definiten Artikel und D-Pronomen des Deutschen.

Bybee, Joan (1985): Morphology. A Study of Relation between Meaning and Form. Amsterdam: Benjamins.

Bybee, Joan (1988): Morphology as lexical organisation. In Michael Hammond \& Michael Noonan (Hrsg.), Theoretical Morphology. Approaches in Modern Linguistics, 119-141. San Diego: Academic Press.

Bybee, Joan (2010): Language, Usage and Cognition. Cambridge: Cambridge University Press.

Casenhiser, Devin \& Adele Goldberg (2005): Fast mapping between a phrasal form and meaning. Developmental Science 8(6), 500-508.

Cristante, Valentina (2016): The Processing of Non-Canonical Sentences in Children with German as a First or Second Language and German Adults. Evidence from an EyeTracking Study. Dissertation, Universität Münster. http://nbn-resolving.de/urn:nbn:de: hbz:6-11269715348 (05.05.2020).

Cristante, Valentina \& Sarah Schimke (2018): Die Verarbeitung von Passivsätzen und OVS-Sätzen im kindlichen L2-Erwerb. In Sarh Schimke \& Holger Hopp (Hrsg.), 
Sprachverarbeitung im Zweitspracherwerb, 169-192. Berlin, Boston: De Gruyter Mouton.

Dittmar, Miriam, Kirsten Abbot-Smith, Elena Lieven \& Michael Tomasello (2008): German children's comprehension of word order and case marking in causative sentences. Child Development 79(4), 1152-1167.

Draye, Luk (2002): Aspects of nominative and accusative in German. In Kristin Davidse \& Béatrice Lamiroy (Hrsg.), The Nominative and Accusative and their Counterparts, 175-200. Amsterdam, Philadelphia: Benjamins.

Dryer, Matthew S. (2013): Order of Subject, Object and Verb. In Matthew S. Dryer \& Martin Haspelmath (Hrsg.), The World Atlas of Language Structures Online. Leipzig: Max Planck Institute for Evolutionary Anthropology. http://wals.info/chapter/81 (28.03.2019).

Duden (2016) = Duden. Die Grammatik. 9., vollständig überarbeitete und aktualisierte Aufl. Hrsg. von Angelika Wöllstein und der Dudenredaktion. Berlin: Dudenverlag (Der Duden 4).

Dürscheid, Christa (2007): Quo vadis, Casus? Zur Entwicklung der Kasusmarkierung im Deutschen. In Hartimut E. H. Lenk \& Maik Walter (Hrsg.), Wahlverwandtschaften. Valenzen - Verben - Varietäten. Festschrift für Klaus Welke zum 70. Geburtstag, 89-112. Hildesheim: Georg Olms.

Eisenberg, Peter (2013): Grundriss der deutschen Grammatik. Band 2: Der Satz. 4., aktualisierte und überarbeite Aufl. Stuttgart: Metzler.

Erlam, Rosemary (2006): Elicited Imitation as a Measure of L2 Implicit Knowledge: An Empirical Validation Study. Applied Linguistics 27(3), 464-491.

Fengler, Anja, Lars Meyer \& Angela D. Friederici (2015): Brain structural correlates of complex sentence comprehensionin children. Developmental Cognitive Neuroscience (15) 2015, 48-57.

Gamper, Jana (2016): Satzinterpretationsstrategien mehr- und einsprachiger Kinder im Deutschen. Tübingen: Narr.

Gamper, Jana, Verena Wecker \& Carsten Szardenings (i. d. B.): Genus vor Plural: Zum Zusammenhang der nominalen Kategorien im ein- und mehrsprachigen Erwerb.

Göksel, Aslı \& Celia Kerslake (2011): Turkish. A Comprehensive Grammar. London, New York: Routledge. https://www.ncbi.nlm.nih.gov/pmc/articles/PMC4773164/pdf/pone. 0149870.pdf, (28.03.2019).

Grießhaber, Wilhelm (i. d. B.): Muster und Frequenz: Nominalflexion nach Sprachkenntnissen und Sprachstatus.

Indefrey, Peter (2002): Listen und Regeln. Erwerb und Repräsentation der schwachen Substantivdeklination des Deutschen. Dissertation, Heinrich-Heine-Universität Düsseldorf.

Kempe, Vera \& Brian MacWhinney (1998): The acquisition of case marking by adult learners of Russian and German. Studies in Second Language Acquisition 20, 543-587.

Köpcke, Klaus-Michael (1993): Schemata bei der Pluralbildung im Deutschen. Versuch einer kognitiven Morphologie. Tübingen: Narr.

Köpcke, Klaus-Michael (1995): Die Klassifikation der schwachen Maskulina in der deutschen Gegenwartssprache. Ein Beispiel für die Leistungsfähigkeit der Prototypentheorie. Zeitschrift für Sprachwissenschaft 14, 159-180.

Köpcke, Klaus-Michael (2005): ,Die Prinzessin küsst den Prinz“ - Fehler oder gelebter Sprachwandel? Didaktik Deutsch 18, 67-83. 
Kürschner, Sebastian (i.d.B.): Schemata im Wandel der schwedischen und dänischen maskulinen Deklinationsklassen - ein Vergleich des Deutschen mit nah verwandten Sprachen.

MacWhinney, Brian (2001): The Competition Model: the input, the context, and the Brain. In Peter Robinson (Hrsg.), Cognition and Second Language Instruction, 69-90. Cambridge: Cambridge University Press.

MacWhinney, Brian (2017): A unified model of first and second language learning. In Maya Hickmann, Edy Veneziano \& Harriet Jisa (Hrsg.), Sources of Variation in First Language Acquisition: Languages, Contexts, and Learners, 287-312. Amsterdam: John Benjamins.

Musan, Renate (2010): Informationsstruktur. Kurze Einführungen in die germanistische Linguistik. Heidelberg: Winter.

Nesset, Tore (2008): Abstract Phonology in a Concrete Model. Cognitive Linguistics and the Morphology-Phonology Interface. Berlin, New York: De Gruyter.

Paulfranz, Alexandra (2013): Kasusmarkierungen der Gegenwartssprache in deutschen Lokalund Regionaltageszeitungen. University of Bamberg Press.

Pregel, Dietrich \& Gert Rickheit (1987): Der Wortschatz im Grundschulalter. Häufigkeitswörterbuch zum verbalen, substantivischen und adjektivischen Wortgebrauch. Hildesheim: Olms.

Primus, Beatrice (1999): Case and Thematic Roles: Ergative, Accusative and Active. Niemeyer: Tübingen.

Roesch, Anne-Dorothee \& Vicky Chondrogianni (2014): Morphological cues in the comprehension of wh-questions in German-speaking simultaneous and early sequential bilingual children. In Will Orman \& Matthew J. Valleau (Hrsg.), Proceedings of the 38th Boston University Conference on Language Development (BUCLD 38), 380-392. Somerville, MA: Cascadilla Press.

Roesch, Anne-Dorothee \& Vicky Chondrogianni (2015): The use of case in the comprehension of wh-questions in German-speaking children with and without SLI. In Cornelia Hamann \& Esther Ruigendijk (Hrsg.): Proceedings of GALA 2013, 379-402. Cambridge: Cambridge Scholars.

Roesch, Anne-Dorothee \& Vicky Chondrogianni (2016): 'Which mouse kissed the frog?': Effects of age of onset, length of exposure and knowledge of case-marking on the comprehension of wh-questions in German-speaking simultaneous and early sequential bilingual children. Journal of Child Language 43, 635-661.

Ronneberger-Sibold, Elke (i.d. B.): Wie dekliniert man der Truntáke in Bayern? Schemata und Prototypen in der Deklination von Kunstwörtern in verschiedenen deutschen Varietäten.

Schipke, Christine S., Lisa J. Knoll, Angela D. Friederici \& Regine Oberecker (2012): Preschool children's interpretation of object-initial sentences: neural correlates of their behavioral performance. Developmental Science 15, 762-774.

Schouwenaars, Atty, Petra Hendriks \& Esther Ruigendijk (2018): German children's processing of morphosyntactic cues in wh-questions. Applied Psycholinguistics, 1-40.

Schroeder, Sascha, Kay-Michael Würzner, Julian Heister, Alexander Geyken \& Reinhold Kliegl (2015): childLex: Eine lexikalische Datenbank zur Schriftsprache für Kinder im Deutschen. Psychologische Rundschau, 66, 155-165. doi:10.1026/0033-3042/a000275, 
https://www.mpib-berlin.mpg.de/sites/default/files/media/pdf/409/childlex_rund schau_3rdrevision_2015-01-27.pdf (02.04.2019).

Thieroff, Rolf (2003): Die Bedienung des Automatens durch den Mensch. Deklination der schwachen Maskulina als Zweifelsfall. Linguistik online 16, 4/03. https://www.linguistikonline.net/16_03/thieroff.html (28.03.2019).

Wecker, Verena (2016): Strategien bei der Pluralbildung im DaZ-Erwerb. Eine Studie mit russisch- und türkischsprachigen Lernern. Berlin, Boston: De Gruyter. 
